\title{
Diagnostic value of molybdenum target combined with DCE-MRI in different types of breast cancer
}

\author{
YING HU, YAN ZHANG and JINGLIANG CHENG \\ Department of MRI, The First Affiliated Hospital of Zhengzhou University, Zhengzhou, Henan 450000, P.R. China
}

Received May 7, 2019; Accepted June 26, 2019

DOI: 10.3892/ol.2019.10746

\begin{abstract}
This study compared the diagnostic value of molybdenum target and dynamic contrast-enhanced magnetic resonance imaging (DCE-MRI) in different types of breast cancer. A retrospective analysis was performed on 120 female patients with breast cancer admitted to The First Affiliated Hospital of Zhengzhou University from February 2015 to October 2017. All of them underwent DCE-MRI and molybdenum target examination. Postoperatively, the pathological tissues were examined to determine their molecular typing. The sensitivity and diagnostic coincidence rates of Luminal-B breast cancer with different molecular types diagnosed by molybdenum target combined with DCE-MRI were significantly higher than those of molybdenum target or DCE-MRI alone. There were no statistical differences in sensitivity and diagnostic coincidence rates of breast cancer with different molecular types diagnosed by molybdenum target or DCE-MRI alone between the two groups $(\mathrm{P}>0.05)$. Molybdenum target combined with DCE-MRI in the diagnosis of different molecular types of breast cancer is better than the single imaging screening, which is of great clinical significance in the development of individualized comprehensive treatment for breast cancer patients and worthy of wide promotion in clinical practice.
\end{abstract}

\section{Introduction}

Breast cancer tends to occur in the epithelial tissue of the breast gland and is one of the most common female malignant tumors (1). Breast cancer has a great impact on women's physical and mental health, and in severe cases, it can even be life-threatening. According to statistics (2), the incidence of breast cancer worldwide is on the rise year by year (3). Since most surgical patients are already in the advanced stage of breast

Correspondence to: Dr Yan Zhang, Department of MRI, The First Affiliated Hospital of Zhengzhou University, 1 Jianshe East Road, Zhengzhou, Henan 450000, P.R. China

E-mail: dvbp31@163.com

Key words: breast cancer typing; molybdenum target, dynamic contrast-enhanced magnetic resonance imaging, diagnostic value cancer when they visit the doctor, even if the cancerous tissue is removed, breast cancer has a high probability to metastasize (4). Statistical data show that the postoperative recurrence rate of breast cancer patients is high and the five-year survival rate is low at this stage (5). Clinical etiology of breast cancer is not completely clear, so early diagnosis of breast cancer helps patients get timely treatment, thus improving the survival rate of breast cancer patients (6). According to relevant reports, Luminal-A, Luminal-B, Her2-overexpressed and triplenegative breast cancers are four different molecular types, and different molecular types are more conducive for clinicians to choose the best individualized treatment according to the characteristics of different molecular sub-types (7). However, molecular typing depends on relevant pathological tissues. Although pathological tissue diagnosis is the gold standard for clinical staging diagnosis of breast cancer, some patients are still psychologically and physiologically unacceptable (8). With the continuous development and innovation of medical diagnostic technology, imaging technology for clinical diagnosis is also continuously upgraded, and the results of diagnostic coincidence rate and pathological tissue diagnosis are getting increasingly closer (9).

At present, molybdenum target imaging (10), breast ultrasound (11), magnetic resonance imaging (MRI) (12), dynamic contrast-enhanced magnetic resonance imaging (DCE-MRI) (13) and positron emission tomography (PET) (14) are new imaging techniques commonly used in clinical diagnosis. However, the breast molybdenum target and other new imaging techniques of DCE-MRI have different limitations (15). Therefore, this study was performed to investigate the clinical efficacy of molybdenum target, DCE-MRI and molybdenum target combined with DCE-MRI in the diagnosis of breast cancer of different types.

\section{Patients and methods}

Data collection of the patients. From February 2015 to October 2017, 120 female patients with breast cancer admitted to The First Affiliated Hospital of Zhengzhou University (Zhengzhou, China) were diagnosed with breast cancer through surgery or pathological biopsy, with the age range of 28-67 years and the mean age of $47.46 \pm 4.54$ years.

The inclusion and exclusion criteria were: i) Only breast cancer patients admitted to The First Affiliated Hospital of Zhengzhou University were included, and all tissue samples 
A

B
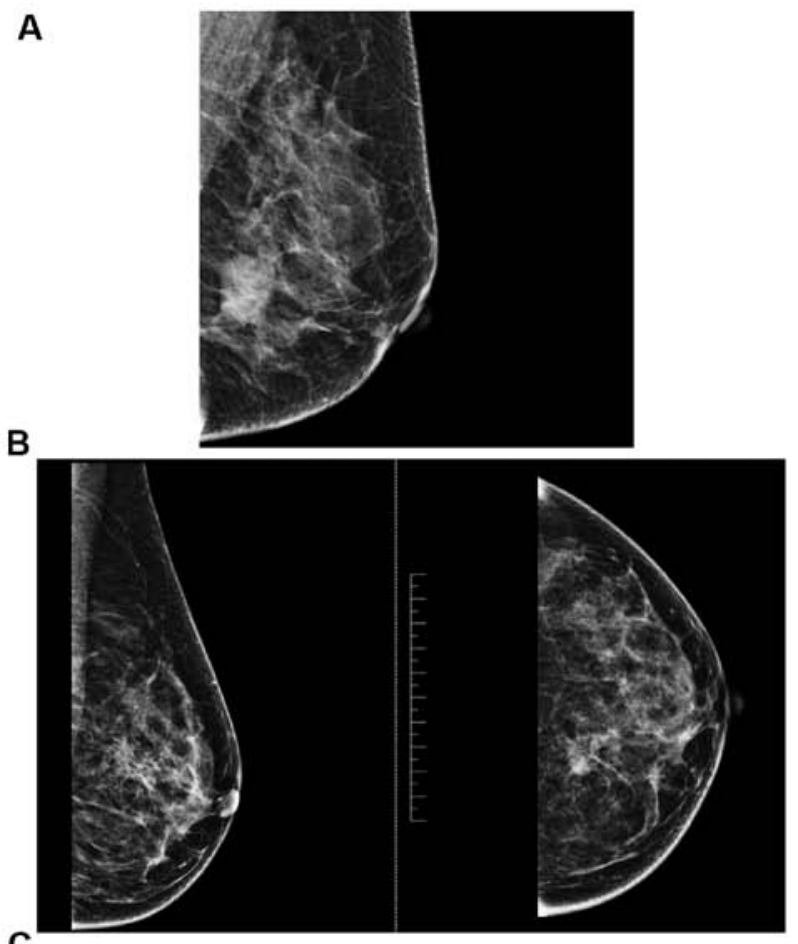

C

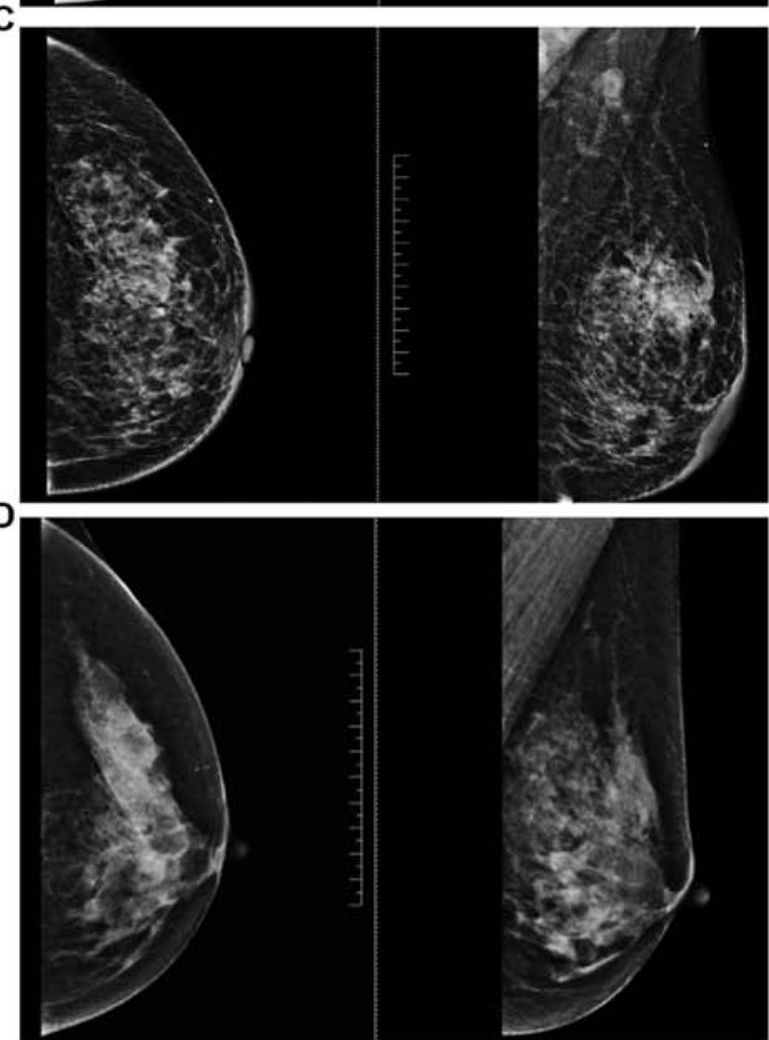

Figure 1. Image features of molybdenum target for different types of breast cancer. (A) Luminal-A breast cancer. Molybdenum targets are mostly burr masses with irregular edges. An infiltrating carcinoma of the left breast and non-special type, Luminal-A breast cancer. (B) Luminal-B breast cancer. The molybdenum target of breast cancer is usually a marginal irregular mass, and the distortion of structure is common when the mass is not obvious. An infiltrating breast cancer and non-special type, Luminal-B breast cancer. (C) Her2-overexpressed breast cancer. The molybdenum target of breast cancer is usually a mass with fuzzy edges, and the cluster-like microcalcification is more common. An infiltrating carcinoma of the right breast and non-special type, Her2-overexpressed breast cancer. (D) Triple-negative breast cancer. The molybdenum target of breast cancer is usually a large mass with smooth edges, and there are few calcification and structural distortion. An infiltrating carcinoma and intraductal carcinoma of the left breast, triplenegative breast cancer.

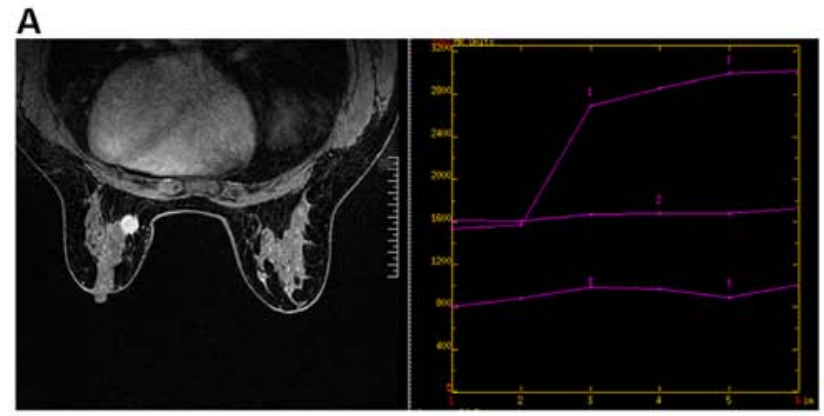

B

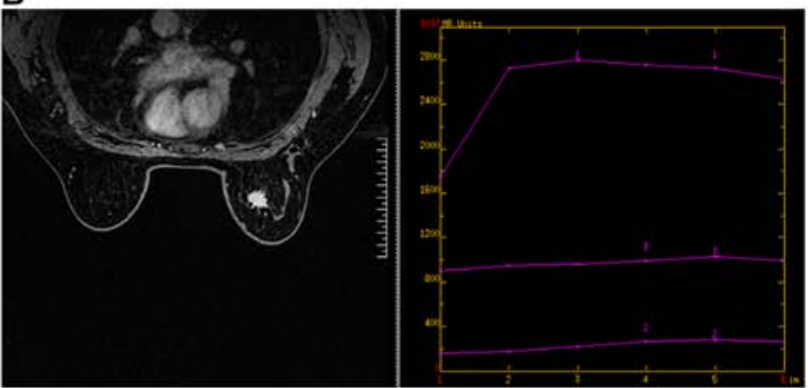

C

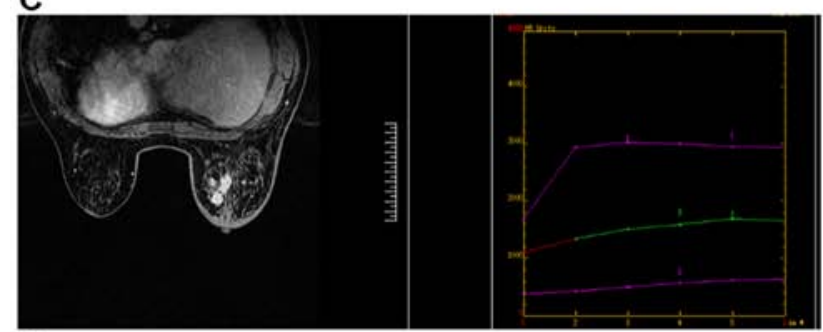

$\bar{D}$

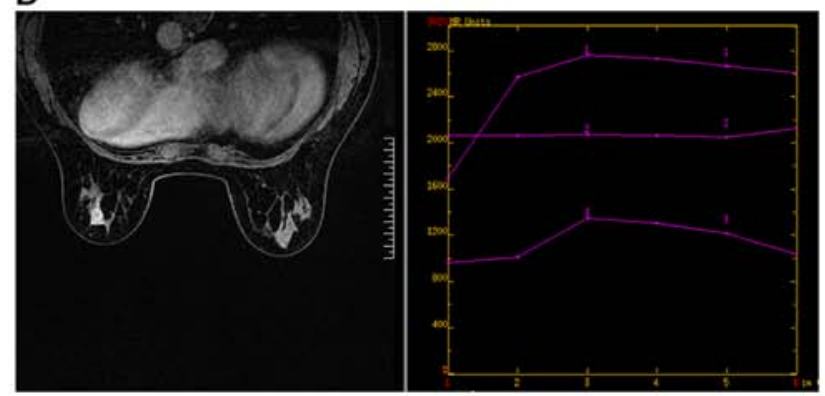

Figure 2. DCE-MRI image features of different types of breast cancer. (A) Luminal-A breast cancer. DCE-MRI shows a larger mass with smooth edges, less calcification and structural distortion. Irregular mass shadow can be seen at about 9 o'clock in the left breast. The lesion margin is rough with visible bristles, and the signal shadow is equal to T1 mixed with long T2. The dispersion of DWI is limited and shows uneven enhancement, and the time signal intensity curve shows an inflow pattern. (B) Luminal B breast cancer. DCE-MRI shows most uneven enhancement in the lesion area with burrs on the margin. Irregular mass shadow can be seen at about 1 o'clock in the right breast, with multiple bristles on the edge, showing equal $\mathrm{T} 1$ and long $\mathrm{T} 2$ signal shadow. The dispersion of DWI is limited and shows uneven enhancement, and the time signal intensity curve shows an outflow pattern. (C) Her2 overexpressed breast cancer. DCE-MRI shows dynamic enhancement of the lesion area. Multiple clumps and focal distribution lesions can be seen in the upper and lower quadrant of the right breast, showing equal/slightly longer T1 mixed with slightly longer T2 signals. DWI has limited dispersion and unclear boundary. There are burrs and uneven enhancement at the edge of the mass in the outer upper quadrant of the right breast. The curve of time signal intensity is plateau, and multiple lymph node shadows are seen in the right axilla. (D) Triple-negative breast cancer. DCE-MRI shows ring enhancement in the lesion area. Irregular mass shadow can be seen at 5 o'clock in the left breast, showing mixed t1-long and t2-long signal shadows. The non-uniform annular dispersion of DWI is limited, and the non-uniform annular enhancement is enhanced. Burrs are visible at the edges, and the time signal intensity curve presents an outflow pattern. DCE-MRI, dynamic contrast-enhanced magnetic resonance imaging. 
were diagnosed as breast cancer after joint examination by general surgery and pathology department (16). No radiotherapy and chemotherapy or other treatment was given. ii) Pregnant women and patients with allergic reaction, claustrophobia and other contraindications to contrast media were excluded. Informed consent forms were signed in advance by patients and their families.

The study was approved by the Ethics Committee of the First Affiliated Hospital of Zhengzhou University.

Instruments and methods. GE Seno molybdenum target mammography machine (purchased from Shenzhen Mercery Electronics Co., Ltd.) was used. Automatic parameters were selected and X-ray exposure was automatically adjusted according to the density of mammary glands. All patients underwent imagings in double nipple caudal position (CC position) and mediolateral oblique (MLO position), with breast being moderately squeezed from left and right. DCE-MRI (Siemens) scanning was performed in 3DT1-weighted sequence axial, with a total of 6 phases (the first phase was plain scanning of the mask, and the following 5 phases were enhanced scanning). Scan parameters: TR/TE: $4.32 / 1.57 \mathrm{msec}$, double Angle (FA) $10^{\circ}$, FOV: $34 \times 34 \mathrm{~cm}$, matrix: $448 \times 448$, incentive number: 1 time, layer thickness: $1 \mathrm{~mm}$; there was no distance between scan, each scan lasted $1 \mathrm{~min}$ and $7 \mathrm{sec}$, with a total scanning time of $7 \mathrm{~min}$ and $2 \mathrm{sec}$. The contrast enhancement agent gadolinium Gd-DTPA (purchased from ACCDON Inc.) was injected with a dose of $0.1 \mathrm{mmol} / \mathrm{kg}$ and a rate of $3.0 \mathrm{ml} / \mathrm{sec}$ through the elbow vein.

Significant image features of breast cancer with different types of molybdenum target and DCE-MRI. The significant image features of breast cancer with different types of molybdenum target and DCE-MRI are shown in Figs. 1 and 2.

Statistical analysis. Application of SPSS 17.0 (Beijing Boyizhixun Information Technology Co., Ltd.) software system was used for statistical analysis and $\chi^{2}$ inspection for comparison of accuracy rate of diagnosis. Enumeration data were expressed as [n (\%)]. $\mathrm{P}<0.05$ was considered to indicate a statistically significant difference.

\section{Results}

General data. General clinical data of the patients are shown in Table I.

Diagnostic efficacy of molybdenum target, DCE-MRI and their combined detection in the diagnosis of different types of breast cancer

Luminal-A breast cancer. The sensitivity, specificity and diagnostic coincidence rates of Luminal-A breast cancer diagnosed by molybdenum target were $84.00,82.86$ and $83.33 \%$, respectively. The sensitivity, specificity and diagnostic coincidence rates of Luminal-A breast cancer diagnosed by DCE-MRI were $90.00,88.57$ and $89.17 \%$, respectively. The sensitivity, specificity and diagnostic coincidence rates of Luminal-A breast cancer diagnosed by molybdenum target combined with DCE-MRI were 98.00, 88.57 and $92.50 \%$, respectively. The sensitivity and diagnostic coincidence rates
Table I. General clinical data of the patients.

\begin{tabular}{lc}
\hline Factors & {$[\mathrm{n}(\%)]$} \\
\hline Age (years) & \\
$<47.46$ & $48(40.00)$ \\
$\geq 47.46$ & $72(60.00)$ \\
Smoking & \\
Yes & $84(70.00)$ \\
No & $36(30.00)$ \\
Alcohol consumption & \\
Yes & $65(54.17)$ \\
No & $55(45.83)$ \\
Menopausal status & \\
Premenopause & $80(66.67)$ \\
Post-menopause & $40(33.33)$ \\
Differentiated degree & \\
High & $51(42.50)$ \\
Middle & $30(25.00)$ \\
Low & $39(32.50)$ \\
Lymphatic metastasis & \\
Yes & $39(32.50)$ \\
No & $81(67.50)$ \\
Different classification & \\
Luminal-A type & $50(41.67)$ \\
Luminal-B type & $31(25.83)$ \\
Her2-overexpressed type & $20(16.67)$ \\
Triple-negative type & $19(15.83)$
\end{tabular}

of Luminal-A breast cancer diagnosed by molybdenum target combined with DCE-MRI were significantly higher than those of Luminal-A breast cancer diagnosed by molybdenum target or DCE-MRI alone. There was no statistical difference in sensitivity and diagnostic coincidence rates of Luminal-A breast cancer diagnosed by molybdenum target or DCE-MRI alone $(\mathrm{P}>0.05)$ (Tables II and III).

Luminal-B breast cancer. The sensitivity, specificity and diagnostic coincidence rates of Luminal-B breast cancer diagnosed by molybdenum target were $80.65,82.02$ and $81.67 \%$, respectively. The sensitivity, specificity and diagnostic coincidence rate of Luminal-B breast cancer diagnosed by DCE-MRI were $87.10,87.64$ and $87.50 \%$, respectively. The sensitivity, specificity and diagnostic coincidence rates of Luminal-B breast cancer diagnosed by molybdenum target combined with DCE-MRI were 96.77, 86.51 and $89.17 \%$, respectively. The sensitivity and diagnostic coincidence rate of Luminal-B breast cancer diagnosed by molybdenum target combined with DCE-MRI were significantly higher than those of Luminal-B breast cancer diagnosed by molybdenum target or DCE-MRI alone. There was no statistical difference in sensitivity and diagnostic coincidence rates of Luminal-B breast cancer diagnosed by molybdenum target or DCE-MRI alone ( $\mathrm{P}>0.05)$ (Tables IV and V).

Her2-overexpressed breast cancer. The sensitivity, specificity and diagnostic coincidence rates of Her2-overexpressed 
Table II. Results of Luminal-A breast cancer diagnosed by different method.

\begin{tabular}{llrrr}
\hline \multirow{2}{*}{ Diagnosis methods } & \multicolumn{2}{c}{ Pathological results } & \\
\cline { 3 - 4 } Molybdenum target & \multicolumn{1}{c}{ Group } & Luminal-A type & Non-luminal-A type & Total \\
& Luminal-A type & 42 & 12 & 54 \\
& Non-luminal-A type & 8 & 58 & 66 \\
DCE-MRI & Total & 50 & 70 & 120 \\
& Luminal-A type & 45 & 8 & 53 \\
& Non-luminal-A type & 5 & 62 & 67 \\
Molybdenum target combined & Total & 50 & 70 & 120 \\
with DCE-MRI & Luminal-A type & 49 & 62 & 57 \\
& Non-luminal-A type & 1 & 70 & 120 \\
\hline
\end{tabular}

DCE-MRI, dynamic contrast-enhanced magnetic resonance imaging.

Table III. Diagnostic efficacy of molybdenum target, DCE-MRI and their combined detection in the diagnosis of Luminal-A breast cancer.

\begin{tabular}{|c|c|c|c|c|c|}
\hline Groups & $\begin{array}{l}\text { Molybdenum } \\
\text { target diagnosis }\end{array}$ & $\begin{array}{c}\text { DCE-MRI } \\
\text { diagnosis }\end{array}$ & $\begin{array}{c}\text { Joint } \\
\text { diagnosis }\end{array}$ & $\chi^{2}$ value & P-value \\
\hline Sensitivity & $84.00 \%(42 / 50)$ & $90.00 \%(45 / 50)$ & $98.00 \%(49 / 50)$ & 0.284 & 0.868 \\
\hline Specificity & $82.86 \%(58 / 70)$ & $88.57 \%(62 / 70)$ & $88.57 \%(62 / 70)$ & 0.095 & 0.954 \\
\hline Diagnostic coincidence rate & $83.33 \%(100 / 120)$ & $89.17 \%(107 / 120)$ & $92.50 \%(111 / 120)$ & 0.313 & 0.855 \\
\hline
\end{tabular}

DCE-MRI, dynamic contrast-enhanced magnetic resonance imaging.

Table IV. Results of Luminal-B breast cancer diagnosed by different methods.

\begin{tabular}{|c|c|c|c|c|}
\hline \multirow[b]{2}{*}{ Diagnosis methods } & \multirow[b]{2}{*}{ Group } & \multicolumn{2}{|c|}{ Pathological results } & \multirow[b]{2}{*}{ Total } \\
\hline & & Luminal-B type & Non-luminal-B type & \\
\hline \multirow[t]{3}{*}{ Molybdenum target } & Luminal-B type & 25 & 16 & 41 \\
\hline & Non-luminal-B type & 6 & 73 & 79 \\
\hline & Total & 31 & 89 & 120 \\
\hline \multirow[t]{3}{*}{ DCE-MRI } & Luminal-B type & 27 & 11 & 38 \\
\hline & Non-luminal-B type & 4 & 78 & 82 \\
\hline & Total & 31 & 89 & 120 \\
\hline \multirow{3}{*}{$\begin{array}{l}\text { Molybdenum target combined } \\
\text { with DCE-MRI }\end{array}$} & Luminal-B type & 30 & 12 & 42 \\
\hline & Non-luminal-B type & 1 & 77 & 78 \\
\hline & Total & 31 & 89 & 120 \\
\hline
\end{tabular}

DCE-MRI, dynamic contrast-enhanced magnetic resonance imaging.

breast cancer diagnosed by molybdenum target were 80.00 , 87.00 and $85.83 \%$, respectively. The sensitivity and diagnostic coincidence rates of Her2-overexpressed breast cancer diagnosed by DCE-MRI were $85.00,88.00$ and $87.50 \%$, respectively. The sensitivity, specificity and diagnostic coincidence rates of Her2-overexpressed breast cancer diagnosed by molybdenum target combined with DCE-MRI were 95.00, 88.00 and $89.17 \%$, respectively. The sensitivity, specificity and diagnostic coincidence rates of Her2-overexpressed breast cancer diagnosed by molybdenum target combined with DCE-MRI were significantly higher than those of Her2-overexpressed breast cancer diagnosed by molybdenum target or DCE-MRI 
Table V. Diagnostic efficacy of molybdenum target, DCE-MRI and their combined detection in the diagnosis of Luminal-B breast cancer.

\begin{tabular}{|c|c|c|c|c|c|}
\hline Groups & $\begin{array}{l}\text { Molybdenum } \\
\text { target diagnosis }\end{array}$ & $\begin{array}{l}\text { DCE-MRI } \\
\text { diagnosis }\end{array}$ & Joint diagnosis & $\chi^{2}$ value & P-value \\
\hline Sensitivity & $80.65 \%(25 / 31)$ & $87.10 \%(27 / 31)$ & $96.77 \%(30 / 31)$ & 0.245 & 0.885 \\
\hline Specificity & $82.02 \%(73 / 89)$ & $87.64 \%(78 / 89)$ & $86.51 \%(77 / 89)$ & 0.100 & 0.951 \\
\hline Diagnostic coincidence rate & $81.67 \%(98 / 120)$ & $87.50 \%(105 / 120)$ & $89.17 \%(107 / 120)$ & 0.235 & 0.890 \\
\hline
\end{tabular}

DCE-MRI, dynamic contrast-enhanced magnetic resonance imaging.

Table VI. Results of Her2-overexpressed breast cancer diagnosed by different method.

\begin{tabular}{llrrr}
\hline & & \multicolumn{2}{c}{ Pathological results } \\
\cline { 3 - 4 } Diagnosis methods & \multicolumn{1}{c}{ Group } & $\begin{array}{c}\text { Her2-overexpressed } \\
\text { type }\end{array}$ & $\begin{array}{c}\text { Non-Her2-overexpressed } \\
\text { type }\end{array}$ & Total \\
\hline Molybdenum target & Her2-overexpressed type & 16 & 12 & 28 \\
& Non-Her2-overexpressed type & 4 & 87 & 91 \\
& Total & 20 & 100 & 120 \\
DCE-MRI & Her2-overexpressed type & 17 & 12 & 29 \\
& Non-Her2-overexpressed type & 3 & 88 & 91 \\
Molybdenum target combined & Total & 20 & 100 & 120 \\
with DCE-MRI & Her2-overexpressed type & 19 & 12 & 31 \\
& Non-Her2-overexpressed type & 1 & 88 & 89 \\
& Total & 20 & 100 & 120 \\
\hline
\end{tabular}

DCE-MRI, dynamic contrast-enhanced magnetic resonance imaging.

Table VII. Diagnostic efficacy of molybdenum target, DCE-MRI and their combined detection in the diagnosis of Her2-overexpressed breast cancer.

\begin{tabular}{|c|c|c|c|c|c|}
\hline Groups & $\begin{array}{l}\text { Molybdenum } \\
\text { target diagnosis }\end{array}$ & $\begin{array}{l}\text { DCE-MRI } \\
\text { diagnosis }\end{array}$ & Joint diagnosis & $\chi^{2}$ value & P-value \\
\hline Sensitivity & $80.00 \%(16 / 20)$ & $85.00 \%(17 / 20)$ & $95.00 \%(19 / 20)$ & 0.143 & 0.931 \\
\hline Specificity & $87.00 \%(87 / 100)$ & $88.00 \%(88 / 100)$ & $88.00 \%(88 / 100)$ & 0.004 & 0.998 \\
\hline Diagnostic coincidence rate & $85.83 \%(103 / 120)$ & $87.50 \%(105 / 120)$ & $89.17 \%(107 / 120)$ & 0.041 & 0.980 \\
\hline
\end{tabular}

DCE-MRI, dynamic contrast-enhanced magnetic resonance imaging.

alone. There was no statistical difference in sensitivity and diagnostic coincidence rates of Her2-overexpressed breast cancer diagnosed by molybdenum target or DCE-MRI alone ( $\mathrm{P}>0.05)$ (Tables VI and VII).

Triple-negative breast cancer. The sensitivity, specificity and diagnostic coincidence rates of triple-negative breast cancer diagnosed by molybdenum target were 78.94, 80.20 and $80.00 \%$, respectively. The sensitivity, specificity and diagnostic coincidence rates of triple-negative breast cancer diagnosed by DCE-MRI were $84.21,89.11$ and $88.33 \%$, respectively. The sensitivity, specificity and diagnostic coincidence rates of triple-negative breast cancer diagnosed by molybdenum target combined with DCE-MRI were 94.74, 88.12 and $89.17 \%$, respectively. The sensitivity and diagnostic coincidence rates of triple-negative breast cancer diagnosed by molybdenum target combined with DCE-MRI were significantly higher than those of triple-negative breast cancer diagnosed by molybdenum target or DCE-MRI alone. There was no statistical difference in sensitivity and diagnostic coincidence rates between the two groups in the diagnosis of triple-negative breast cancer with molybdenum target or DCE-MRI alone $(\mathrm{P}>0.05)$ (Tables VIII and IX). 
Table VIII. Results of triple-negative breast cancer diagnosed by different method.

\begin{tabular}{llrrr}
\hline & & \multicolumn{2}{c}{ Pathological results } \\
\cline { 3 - 4 } Diagnosis methods & \multicolumn{1}{c}{ Group } & $\begin{array}{c}\text { Triple-negative } \\
\text { breast cancer }\end{array}$ & $\begin{array}{c}\text { Non-triple-negative } \\
\text { breast cancer }\end{array}$ & Total \\
\hline Molybdenum target & Triple-negative breast cancer & 15 & 20 & 35 \\
& Non-triple-negative breast cancer & 4 & 81 & 85 \\
DCE-MRI & Total & 19 & 101 & 120 \\
& Triple-negative breast cancer & 16 & 11 & 27 \\
Molybdenum target combined & Non-triple-negative breast cancer & 3 & 90 & 93 \\
with DCE-MRI & Total & 19 & 101 & 120 \\
& Triple-negative breast cancer & 18 & 12 & 30 \\
& Non triple-negative breast cancer & 1 & 89 & 90 \\
& Total & 19 & 101 & 120 \\
\hline
\end{tabular}

DCE-MRI, dynamic contrast-enhanced magnetic resonance imaging.

Table IX. Diagnostic efficacy of molybdenum target, DCE-MRI and their combined detection in the diagnosis of triple-negative breast cancer.

\begin{tabular}{llllll}
\hline Groups & $\begin{array}{c}\text { Molybdenum } \\
\text { target diagnosis }\end{array}$ & $\begin{array}{c}\text { DCE-MRI } \\
\text { diagnosis }\end{array}$ & Joint diagnosis & $\chi^{2}$ value & P-value \\
\hline Sensitivity & $78.94 \%(15 / 19)$ & $84.21 \%(16 / 19)$ & $94.74 \%(18 / 19)$ & 0.152 & 0.927 \\
Specificity & $80.20 \%(81 / 101)$ & $89.11 \%(90 / 101)$ & $88.12 \%(89 / 101)$ & 0.307 & 0.858 \\
Diagnostic coincidence rate & $80.00 \%(96 / 120)$ & $88.33 \%(106 / 120)$ & $89.17 \%(107 / 120)$ & 0.393 & 0.822 \\
\hline
\end{tabular}

DCE-MRI, dynamic contrast-enhanced magnetic resonance imaging.

\section{Discussion}

The morbidity and mortality of breast cancer are increasing year by year (17). Since the mechanism of breast cancer cannot be clearly explained at present, the key to reduce morbidity and mortality is to accurately diagnose the conditions of breast cancer patients and provide corresponding treatment schemes (18). Medically, specific genotypes are made according to the gene level of breast cancer patients, and treatment plans are made according to different molecular genotypes of breast cancer. Treatment plans and prognosis of breast cancer patients with different molecular genotypes are greatly different $(19,20)$. In this study, molybdenum target, DCE-MRI and molybdenum target combined with DCE-MRI were performed on the patients, respectively, and the examination results were compared with the pathological examination results of the patients, in order to investigate the clinical efficacy of molybdenum target, DCE-MRI and molybdenum target combined with DCE-MRI with different types of breast cancer. First, we analyzed the diagnostic efficacy of molybdenum target, DCE-MRI and their combined detection in the diagnosis of Luminal-A and Luminal-B breast cancer, and found that the sensitivity and diagnostic coincidence rates of Luminal-A and Luminal-B breast cancer were significantly higher than those of molybdenum target or DCE-MRI alone.
There were no statistical differences in sensitivity and diagnostic coincidence rates between Luminal-A and Luminal-B breast cancer diagnosed by molybdenum target or DCE-MRI alone. Luminal-A type is common in early breast cancer with low recurrence rate (21), while Luminal-B type with high histological grade is common in older breast cancer patients (22).

It has been reported that there is a certain correlation between histopathology, molecular biology and related imaging features of tumors, indicating that breast cancer with different molecular types has different imaging manifestations (23). However, in the study of Goffin et al (24) on breast cancer diagnosed by molybdenum target combined with DCE-MRI, it was found that the diagnostic coincidence rates of breast cancer diagnosed by molybdenum target combined with DCE-MRI were significantly higher than those of breast cancer diagnosed by molybdenum target or DCE-MRI alone. This is similar to the research results of this study, which to some extent supports our results. Then, we analyzed the diagnostic efficacy of molybdenum target, DCE-MRI, and molybdenum target combined with DCE-MRI in Her2-overexpressed type and triple-negative breast cancer, and found that the sensitivity and diagnostic coincidence rates of Her2-overexpressed type and triple-negative breast cancer diagnosed by molybdenum target combined with DCE-MRI were apparently higher than those of diagnosis with molybdenum target or DCE-MRI alone. There 
was no statistical difference in the sensitivity and diagnostic coincidence rate between the two groups of Her2-overexpressed breast cancer and triple-negative breast cancer diagnosed by molybdenum target or DCE-MRI alone. It is reported that although molybdenum target is widely used in the screening of breast lesions, it shows poor sensitivity to small lesions located at the edge of the breast, and the sensitivity of molybdenum target to very dense breasts decreases by $>40 \%$ (25). DCE-MRI, applied to the examination of breast cancer, can better show the hemodynamic characteristics of small breast cancer lesions, but it also has a certain rate of missed diagnosis for small sand-like calcification lesions (26). Clinical studies on tumor lesions detected by DCE-MRI combined with molybdenum target showed that DCE-MRI combined with molybdenum target could significantly improve the clinical diagnosis rate (27). Kriege et al (28) also carried out DCE-MRI, molybdenum target and DCE-MRI combined with molybdenum target detection for breast cancer with different molecular types, and also found that the diagnostic coincidence rates of DCE-MRI combined with molybdenum target for breast cancer were significantly higher than those of the single imaging detection.

In this study, due to the regional limitations of the inclusion of research objects, the experimental results may be biased to some extent. Therefore, we will continue to expand the number of subjects in different regions for this research, and conduct follow-up.

Collectively, the diagnostic efficacy of molybdenum target combined with DCE-MRI in breast cancer with different molecular types is better than that of imaging screening alone, which is of great clinical significance in the development of individualized comprehensive treatment for breast cancer patients and is worthy of wide promotion in clinical practice.

\section{Acknowledgements}

Not applicable.

\section{Funding}

No funding was received.

\section{Availability of data and materials}

The datasets used and/or analyzed during the present study are available from the corresponding author on reasonable request.

\section{Authors' contributions}

YH wrote the manuscript. YH and YZ collected and analyzed the general data of patients. JC was responsible for the analysis and discussion of the data. All authors read and approved the final manuscript.

\section{Ethics approval and consent to participate}

The study was approved by the Ethics Committee of the First Affiliated Hospital of Zhengzhou University (Zhengzhou, China). Patients who participated in this research had complete clinical data. Informed consent forms were signed in advance by the patients and their families.

\section{Patient consent for publication}

Not applicable.

\section{Competing interests}

The authors declare that they have no competing interests.

\section{References}

1. Lantz PM and Booth KM: The social construction of the breast cancer epidemic. Soc Sci Med 46: 907-918, 1998.

2. Vallance JK, Courneya KS, Plotnikoff RC and Mackey JR: Analyzing theoretical mechanisms of physical activity behavior change in breast cancer survivors: Results from the activity promotion (ACTION) trial. Ann Behav Med 35: 150-158, 2008.

3. DeSantis CE, Bray F, Ferlay J, Lortet-Tieulent J, Anderson BO and Jemal A: International variation in female breast cancer incidence and mortality rates. Cancer Epidemiol Biomarkers Prev 24: 1495-1506, 2015.

4. Kitamura T, Qian BZ, Soong D, Cassetta L, Noy R, Sugano G, Kato Y, Li J and Pollard JW: CCL2-induced chemokine cascade promotes breast cancer metastasis by enhancing retention of metastasis-associated macrophages. J Exp Med 212: 1043-1059, 2015.

5. Chee W, Lee Y, Im EO, Chee E, Tsai HM, Nishigaki M, Yeo SA, Schapira MM and Mao JJ: A culturally tailored Internet cancer support group for Asian American breast cancer survivors: A randomized controlled pilot intervention study. J Telemed Telecare 23: 618-626, 2017

6. Stuart-Harris R, Dahlstrom JE, Gupta R, Zhang Y, Craft P and Shadbolt B: Recurrence in early breast cancer: Analysis of data from 3,765 Australian women treated between 1997 and 2015. Breast 44: 153-159, 2019.

7. Rouzier R, Perou CM, Symmans WF, Ibrahim N, Cristofanilli M, Anderson K, Hess KR, Stec J, Ayers M, Wagner P, et al: Breast cancer molecular subtypes respond differently to preoperative chemotherapy. Clin Cancer Res 11: 5678-5685, 2005.

8. Edenfield J, Schammel C, Collins J, Schammel D and Edenfield WJ: Metaplastic breast cancer: Molecular typing and identification of potential targeted therapies at a single institution. Clin Breast Cancer 17: e1-e10, 2017.

9. Chou R, Cuevas C, Fu R, Devine B, Wasson N, Ginsburg A, Zakher B, Pappas M, Graham E and Sullivan SD: Imaging techniques for the diagnosis of hepatocellular carcinoma: A systematic review and meta-analysis. Ann Intern Med 162: 697-711, 2015.

10. Ohuchi N, Suzuki A, Sobue T, Kawai M, Yamamoto S, Zheng YF, Shiono YN, Saito H, Kuriyama S, Tohno E, et al; J-START investigator groups: Sensitivity and specificity of mammography and adjunctive ultrasonography to screen for breast cancer in the Japan Strategic Anti-cancer Randomized Trial (J-START): A randomised controlled trial. Lancet 387: 341-348, 2016.

11. Brem RF, Lenihan MJ, Lieberman J and Torrente J: Screening breast ultrasound: Past, present, and future. AJR Am J Roentgenol 204: 234-240, 2015.

12. Huang Q, Luo Y and Zhang Q: Breast ultrasound image segmentation: A survey. Int J CARS 12: 493-507, 2017.

13. Xia W, Yan Z and Gao X: Volume fractions of DCE-MRI parameter as early predictor of histologic response in soft tissue sarcoma: A feasibility study. Eur J Radiol 95: 228-235, 2017.

14. Perera M, Papa N, Christidis D, Wetherell D, Hofman MS, Murphy DG, Bolton D and Lawrentschuk N: Sensitivity, specificity, and predictors of positive 68Ga-prostate-specific membrane antigen positron emission tomography in advanced prostate cancer: a systematic review and meta-analysis. Eur Urol 70: 926-937, 2016.

15. Zhao H, Zou L, Geng $X$ and Zheng S: Limitations of mammography in the diagnosis of breast diseases compared with ultrasonography: A single-center retrospective analysis of 274 cases. Eur J Med Res 20: 49, 2015.

16. Mavaddat N, Michailidou K, Dennis J, Lush M, Fachal L, Lee A, Tyrer JP, Chen TH, Wang Q, Bolla MK, et al; ABCTB Investigators; kConFab/AOCS Investigators; NBCS Collaborators: Polygenic risk scores for prediction of breast cancer and breast cancer subtypes. Am J Hum Genet 104: 21-34, 2019. 
17. Christiansen $P$, Bjerre K, Ejlertsen B, Jensen MB, Rasmussen BB Lænkholm AV, Kroman N, Ewertz M, Offersen B, Toftdahl DB, et al; Danish Breast Cancer Cooperative Group: Mortality rates among early-stage hormone receptor-positive breast cancer patients: A population-based cohort study in Denmark. J Natl Cancer Inst 103: 1363-1372, 2011.

18. Jafari SH,SaadatpourZ, Salmaninejad A,Momeni F, Mokhtari M, Nahand JS, Rahmati M, Mirzaei H and Kianmehr M: Breast cancer diagnosis: Imaging techniques and biochemical markers. J Cell Physiol 233: 5200-5213, 2018.

19. Montes de Oca R, Gurard-Levin ZA, Berger F, Rehman H, Martel E, Corpet A, de Koning L, Vassias I, Wilson LO, Meseure D, et al: The histone chaperone HJURP is a new independent prognostic marker for luminal A breast carcinoma. Mol Oncol 9: 657-674, 2015.

20. Chen L, Li CI, Tang MT, Porter P, Hill DA, Wiggins CL and Cook LS: Reproductive factors and risk of luminal, HER2-overexpressing, and triple-negative breast cancer among multiethnic women. Cancer Epidemiol Biomarkers Prev 25: 1297-1304, 2016.

21. Gallardo A, Garcia-Valdecasas B, Murata P, Teran R, Lopez L, Barnadas A and Lerma E: Inverse relationship between Ki67 and survival in early luminal breast cancer: Confirmation in a multivariate analysis. Breast Cancer Res Treat 167: 31-37, 2018.

22. Adriaenssens E, Vanhecke E, Saule P, Mougel A, Page A Romon R, Nurcombe V, Le Bourhis X and Hondermarck H: Nerve growth factor is a potential therapeutic target in breast cancer. Cancer Res 68: 346-351, 2008.

23. Li H, Zhu Y, Burnside ES, Huang E, Drukker K, Hoadley KA, Fan C, Conzen SD, Zuley M, Net JM, et al: Quantitative MRI radiomics in the prediction of molecular classifications of breast cancer subtypes in the TCGA/TCIA data set. NPJ Breast Cancer 2: 2, 2016.
24. Goffin J, Chappuis PO, Wong N and Foulkes WD: Re: Magnetic resonance imaging and mammography in women with a hereditary risk of breast cancer. J Natl Cancer Inst 93: 1754-1755, 2001.

25. Shao H, Li B, Zhang X, Xiong Z, Liu Y and Tang G: Comparison of the diagnostic efficiency for breast cancer in Chinese women using mammography, ultrasound, MRI, and different combinations of these imaging modalities. J Xray Sci Technol 21: 283-292, 2013.

26. Shimauchi A, Jansen SA, Abe H, Jaskowiak N, Schmidt RA and Newstead GM: Breast cancers not detected at MRI: Review of false-negative lesions. AJR Am J Roentgenol 194: 1674-1679, 2010.

27. Bäuerle T, Bartling S, Berger M, Schmitt-Gräff A, Hilbig H, Kauczor HU, Delorme S and Kiessling F: Imaging antiangiogenic treatment response with DCE-VCT, DCE-MRI and DWI in an animal model of breast cancer bone metastasis. Eur $\mathrm{J}$ Radiol 73: 280-287, 2010.

28. Kriege M, Brekelmans CT, Boetes C, Besnard PE, Zonderland HM, Obdeijn IM, Manoliu RA, Kok T, Peterse H, Tilanus-Linthorst MM, et al; Magnetic Resonance Imaging Screening Study Group: Efficacy of MRI and mammography for breast-cancer screening in women with a familial or genetic predisposition. N Engl J Med 351: 427-437, 2004.

This work is licensed under a Creative Commons Attribution-NonCommercial-NoDerivatives 4.0 International (CC BY-NC-ND 4.0) License. 